\title{
Structural and electrical properties of amorphous carbon-sulfur composite films
}

\author{
LATHA KUMARI* and S V SUBRAMANYAM \\ Department of Physics, Indian Institute of Science, Bangalore 560 012, India
}

MS received 30 March 2004

\begin{abstract}
In this paper, we discuss the synthesis of carbon-sulfur composite ( $a-\mathrm{C}: \mathrm{S})$ films by vapour phase pyrolysis of maleic anhydride and sulfur. Structural changes in the system are analysed by scanning electron microscopy and powder X-ray diffraction. Microhardness test depicts an increase in the value of hardness with an increase in sulfur concentration. Electrical conductivity of composite samples varies with sulfur concentration. Magnetoresistance (MR) measurements show a drastic increase in the value of MR for the samples prepared at $<\mathbf{9 0 0}^{\circ} \mathrm{C}$. Thermal stability of these samples is analysed by thermogravimetric analysis, which depends on the host structure and the amount of intercalated species.
\end{abstract}

Keywords. Amorphous carbon films; pyrolysis; scanning electron microscopy; electrical properties; thermal analysis.

\section{Introduction}

Diamond-like-carbon (DLC) and amorphous carbon ( $a-\mathrm{C})$ have been studied in detail (Robertson 2002; Silva et al 2002), respectively as a route for hard coatings and novel semiconductors. These films exhibit some of the important features of diamond without the necessity of specialized preparation techniques. DLC covers a range of materials with properties that can vary from those similar to graphite to those approaching natural diamond. Hydrogenfree DLC films also known as amorphous carbon films consist of mainly graphitic $s p^{2}$ carbon matrix containing nm-sized clusters of $s p^{3}$ DLC (Filik et al 2003). In recent years extensive research has been done on the doping of these $a$-C films with donors like nitrogen or phosphorus and acceptors like boron or iodine (Latha et al 2003). The crystalline versions of carbon-nitrogen and carbon-phosphorus compounds may have useful mechanical or electronic properties. Sulfur incorporated into diamond semiconductor acts as $n$-type dopant, which also controls the structural and electrical properties (Sakaguchi et al 1999). Some of the earlier studies demonstrated the induction of shallow donor levels in diamond through the incorporation of either phosphorous or sulfur, but concrete experimental evidence on the potential role of sulfur as an $n$-type donor in diamond is yet to be explored (Gupta et al 2003). Hasegawa et al (1999) demonstrated a clear rectification property of the junction between the sulfur-ion implanted and $p$-type (boron doped) regions of CVD-synthesized diamond, suggesting that the sulfurimplanted region is $n$-type.

Magnetoresistance is a sensitive local probe for investigating the scattering process in disordered metallic sys-

*Author for correspondence tems and is closely related to temperature dependence of conductivity. The effect of magnetic field $(B)$ on the weak localization of electrons is similar to that of inelastic scattering. It suppresses the phase coherence between the partial waves associated with an electron and this reduces the localization, causing the resistance to decrease with magnetic field (negative magnetoresistance). The contribution to magnetoresistance from electron-electron interaction is positive and is predominantly due to Zeeman splitting of the spin-up and spin-down bands. The magnetoresistance is interpreted to be due to distortion of electron orbit at low fields and due to shrinkage of electron wave function at high fields. Magnetoresistance shows a $B^{2}$ dependence at low fields and is temperature dependent, whereas at high fields it is independent of temperature and exhibits a $B^{1 / 2}$ dependence (Dugdale 1995).

The thermal stability of any intercalated system depends on the host structure and the amount of intercalated species. It also depends on how well the guest species are bonded to the host atoms. The stability of the host system is found to decrease with the improving crystallinity or ordering. With increasing ordering, not only intercalation but also deintercalation seems easier (Gupta et al 1995).

In the present work, we discuss the effect of sulfur incorporation on the structural properties of $a-\mathrm{C}$ films. Electrical and magnetoresistance measurements performed on the $a-\mathrm{C}: \mathrm{S}$ films are also discussed. In addition, thermal stability of sulfur incorporated $a-\mathrm{C}$ samples is analysed.

\section{Experimental}

\subsection{Sample preparation}

The $a$-C:S films were prepared by vapour phase pyrolysis of carbon rich organic precursor, maleic anhydride as a 
starting material and sulfur powder as intercalation agent using home-built horizontal double zone furnace set-up. Both the materials were taken in predetermined weight ratios and were pyrolysed at various temperatures (700$900^{\circ} \mathrm{C}$ ). The films deposited on unpolished quartz substrates were annealed in inert gas (argon) atmosphere after preparation to overcome the surface contamination. The films were grey in colour and thickness was found to be 1-2 $\mu \mathrm{m}$. The free standing $a$-C:S films deposited on the inner surface of the preparation quartz tube were more brittle and harder than the $a-\mathrm{C}$ films. The degree of sulfur incorporation, $S / P$ represents the initial weight ratio of sulfur to the precursor $(P)$-maleic anhydride.

\subsection{Sample characterization and measurements}

Scanning electron microscopic analysis (SEM) was carried out on $a$-C:S film coated on quartz substrate with Cambridge Instrument (Model S-360, LINK Pentafet-10000). Powder X-ray diffraction (XRD) measurements were performed on these samples using $\mathrm{CuK}_{\alpha}$ radiation in a Philips X'pert X-ray diffraction system. Microhardness tests were conducted (HMV-2000 Shimadzu) on $a-C$ and $a-C: S$ films (film thickness of the range, $1-2 \mu \mathrm{m}$ ) deposited on polished quartz substrates by microindentation technique.

D.C. electrical conductivity and transverse magnetoresistance measurements were performed on these samples in a liquid helium cryostat (Janis Supervaritemp Cryostat equipped with superconducting magnet) from $300 \mathrm{~K}$ down to $1.3 \mathrm{~K}$ with magnetic field of $0-7 \mathrm{~T}$. The measurements were carried out by using a standard four-probe technique. The sample contacts were made using fine enamelled copper wires as contact leads and silver paste as contact material. The power dissipated into the sample is less than $1 \mu \mathrm{W}$ at low temperatures in order to avoid the heat-induced damage of the sample.

Thermal stability of $a-\mathrm{C}: \mathrm{S}$ samples was analysed using a PYRIS EXSTAR-6000 thermal analyser. The samples were heated from $25-600^{\circ} \mathrm{C}$ at a rate of $10^{\circ} \mathrm{C} / \mathrm{min}$ in the presence of $\mathrm{N}_{2}$ gas atmosphere with a flow rate of $150 \mathrm{ml} /$ min. $\alpha$-Alumina was used as the reference material.

\section{Results and discussion}

\subsection{Structural characterization}

SEM studies were performed on $a-\mathrm{C}: \mathrm{S}$ films deposited on unpolished quartz substrate at $900^{\circ} \mathrm{C}$. Figure 1 gives SEM images of $a-\mathrm{C}: \mathrm{S}$ samples with different $S / P$ values. As is evident from the figure, increase in sulfur concentration accounts for the localized, uniform and dense packing (clustering) of carbon atoms. The average size of these spherical globules is about $0.5-0 \cdot 7 \mu \mathrm{m}$ (magnification, $\sim \times 10^{4}$ ). Many disordered forms of carbon show this kind of globular structure. Sometimes these globules are known to be made up of concentric graphitical shells. As such, increase in sulfur concentration does not account for a large change in size of the single globule. But the increase in aggregate size is observed with sulfur concentration.

XRD patterns of $a-\mathrm{C}$ and $a$-C:S samples prepared at $900^{\circ} \mathrm{C}$ and also pure sulfur are shown in figure 2 . The $a-\mathrm{C}$ $(S / P=0)$ sample shows a broad, intense peak around $25^{\circ}$ and a less intense peak around $43^{\circ}$, which confirms the amorphous nature of the system. In contrast, XRD pattern of $a$-C:S $(S / P=0 \cdot 32)$ sample shows crystalline peaks with amorphous background, which confirms that the sys-
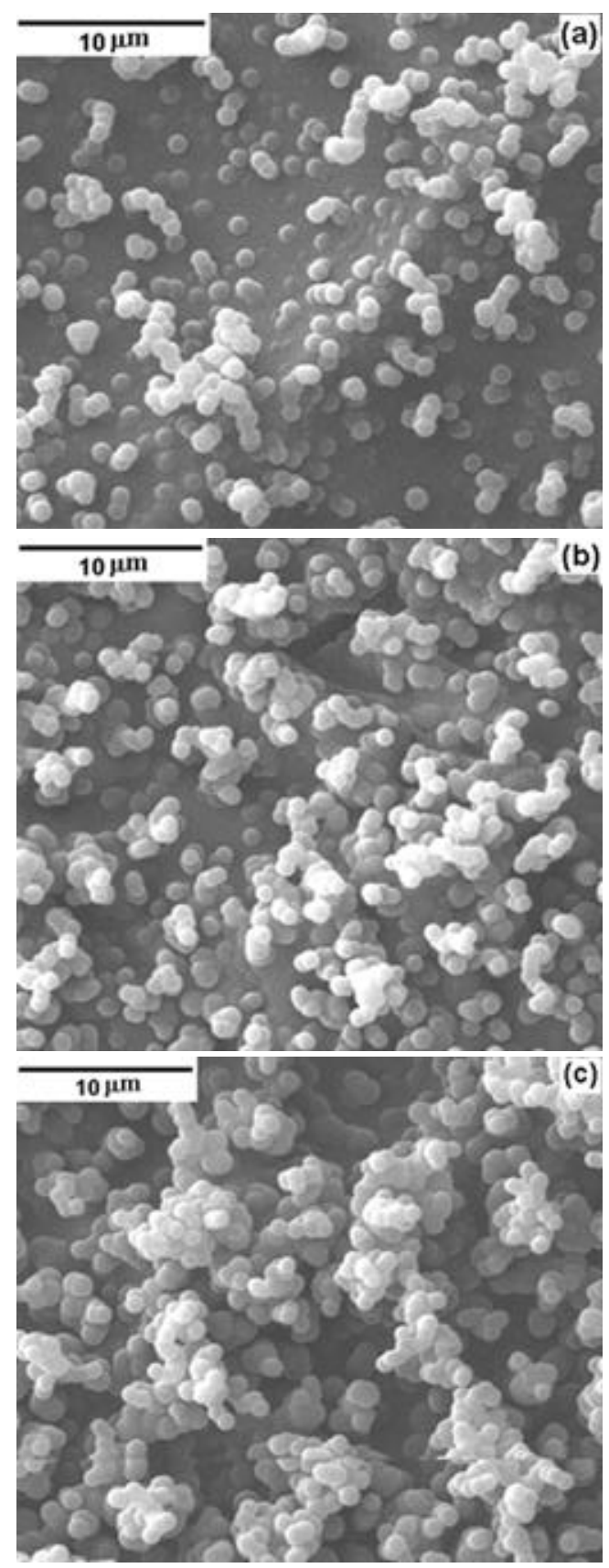

Figure 1. SEM images of $a-\mathrm{C}: \mathrm{S}$ sample prepared at $900^{\circ} \mathrm{C}$ with $S / P$ value of (a) $0 \cdot 16$, (b) 0.32 and (c) 0.96 . 
tem is of two-phase, amorphous and crystalline. These crystalline peaks can be attributed to a carbon-sulfur composite, which corresponds to a sulfur embedded in the carbon matrix. The XRD peaks of $a-\mathrm{C}: \mathrm{S}$ sample are compared with the pure sulfur peaks for confirmation. As is seen from the XRD pattern of $a$-C:S sample, the broad peak at $25^{\circ}$ decreases compared to pure $a$-C and the peak at $43^{\circ}$ totally disappears. These results propose that there is some interaction between sulfur and carbon.

\subsection{Microhardness test}

The microhardness test is conducted on the $a-\mathrm{C}$ and $a$ $\mathrm{C}: \mathrm{S}$ films by indentation technique. The hardness value of the film grown on polished quartz substrate is found by applying a determined load (100-200 g) on the sample and then measuring the diagonally formed pattern. Figure 3 is a comparative plot of the microhardness of the $a-\mathrm{C}$ $(S / P=0)$ and $a-\mathrm{C}: \mathrm{S}(S / P=0 \cdot 16$ and $S / P=0.96)$ samples prepared at $900^{\circ} \mathrm{C}$. The studies depict increase in the value of microhardness with increase in sulfur concentration. Earlier work (Robertson and O'Reilly 1987) showed that, in $a-\mathrm{C}$ a mixture of $s p^{2}$ and $s p^{3}$ carbon sites will tend to segregate into $s p^{2}$-bonded graphitic clusters embedded in a $s p^{3}$-bonded matrix. The $s p^{2}$ clusters were found to control the electronic properties and the connectivity of the $s p^{3}$ matrix largely controls the mechanical properties. A small change in the value of microhardness is observed for the $a$-C:S samples with low sulfur content, but for higher concentration of sulfur, microhardness value increases appreciably. Earlier work on $a-\mathrm{C}$ shows that the increase in the $\mathrm{C}-\mathrm{C}$ coordination value above the critical value, $r_{\mathrm{p}}=2.4$ may account for the finite rigidity and hard-

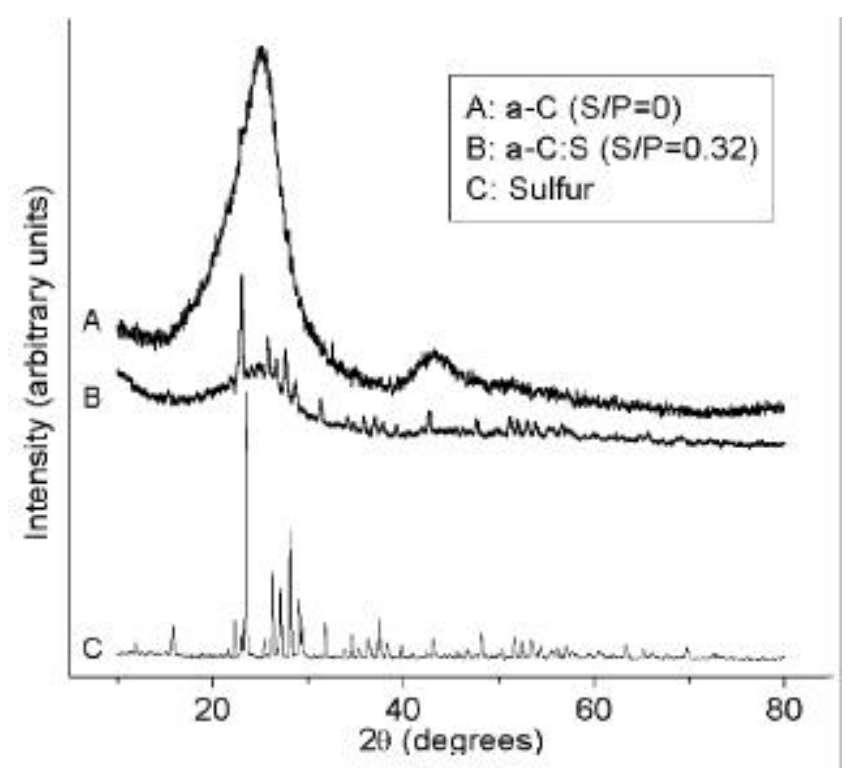

Figure 2. Powder XRD pattern of samples $(S / P=0$ and $S / P=$ 0.32 ) prepared at $900^{\circ} \mathrm{C}$. ness (Robertson 1992). Moreover, the connectivity of the $s p^{3}$ matrix in $a-\mathrm{C}$ system appreciably controls the mechanical properties.

\subsection{Electrical conductivity}

The electrical properties of $a$-C samples depend on the pyrolysis temperature and sulfur content. Figure 4 shows the variation of conductivity as a function of sulfur concentration for samples prepared at various pyrolysis temperatures. Conductivity of the $a-\mathrm{C}: \mathrm{S}$ sample increases with sulfur concentration up to some optimum value of $S / P$ and the conductivity decreases with further addition of sulfur. Moreover, samples prepared at higher pyrolysis temperature and with large sulfur content show a drastic reduction in conductivity. The overall conductivity of the samples may be understood as due to the jumping or tunneling of charge carriers between the remote conductive chains. Appreciable change in conductivity is observed for samples prepared at $900^{\circ} \mathrm{C}$ with sulfur incorporation than for the samples of $700^{\circ} \mathrm{C}$ and $800^{\circ} \mathrm{C}$ preparation. Indeed, the sample of $700^{\circ} \mathrm{C}$ preparation shows very weak dependence of conductivity on sulfur concentration. Since the conductivity depends on the carrier concentration and crystallinity, the relatively low conductivity values are related to low mobility arising from crystal defects and grain boundaries induced by sulfur addition and to low carrier concentrations due to incomplete ionization of $\mathrm{S}$ atoms (Gupta et al 2003). Sulfur incorporated nanocomposite carbon thin films exhibited an increase in conductivity by four orders of magnitude with an increase in sulfur content compared with those grown without sulfur (Gupta et al 2002). Also, earlier work on sulfur incorpo-

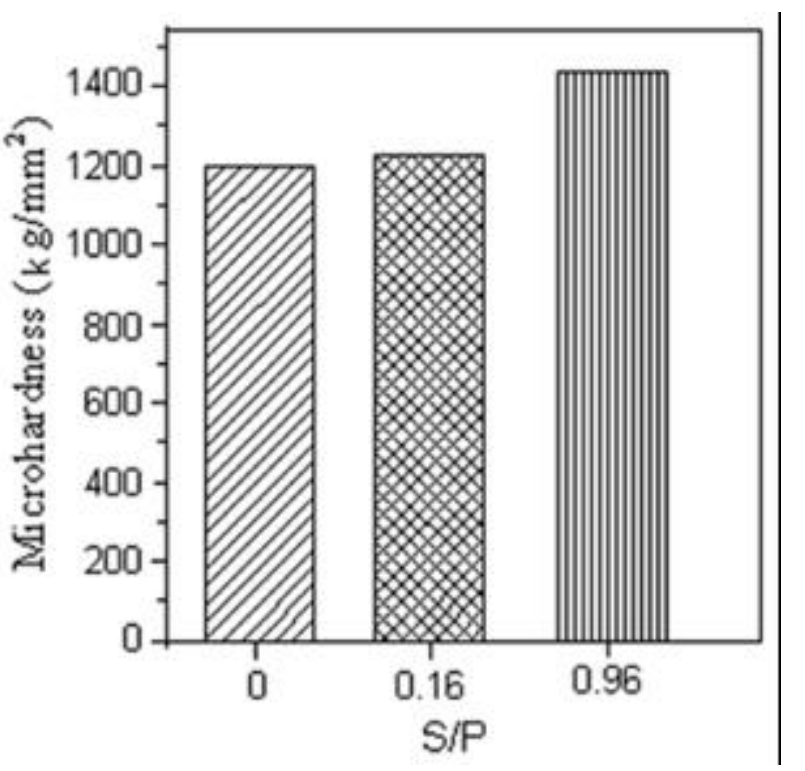

Figure 3. Microhardness of $a-\mathrm{C}$ and $a-\mathrm{C}: \mathrm{S}(S / P=0 \cdot 16$ and $S / P=$ $0.96)$ films. 
rated microcrystalline diamond thin films showed an increase in $n$-type conductivity with increase in sulfur concentration up to moderate value, but for higher sulfur content decrease in conductivity was observed with sign reversal of Hall coefficient (Gupta et al 2003).

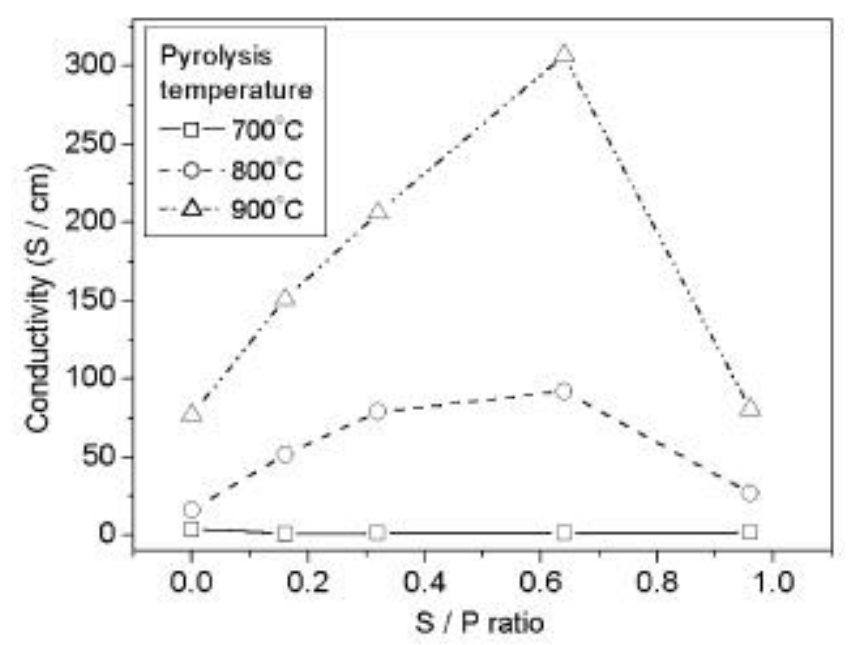

Figure 4. Conductivity at $300 \mathrm{~K}$ vs sulfur content for $a$-C:S samples prepared at different pyrolysis temperatures.

\subsection{Magnetoresistance}

Variation of magnetoresistance, $M R=\left[\left(R_{\mathrm{B}}-R_{0}\right) / R_{0}\right]$ as a function of magnetic field for $a-\mathrm{C}: \mathrm{S}$ samples prepared at $800^{\circ} \mathrm{C}$ with different $S / P$ values is as shown in figure 5 . From the plot, it is evident that magnetoresistance exhibits different behaviours at different temperatures. Samples with $S / P=0.16$ and $S / P=0.32$ show decrease in the value of magnetoresistance than the sample with $S / P=0$, whereas the magnetoresistance increases drastically for samples with $S / P>0.32$ at all temperatures. Even an enormous change in magnetoresistance is observed for $a-C: S$ samples prepared at $700^{\circ} \mathrm{C}$. But the observed change in magnetoresistance is very small for $a-\mathrm{C}: \mathrm{S}$ samples prepared at $900^{\circ} \mathrm{C}$. At temperatures $>10 \mathrm{~K}$, the samples show negative magnetoresistance at low fields, but at higher fields sudden crossover to positive magnetoresistance is accounted. Almost a $6 \%$ increase in the value of magnetoresistance is observed for saturation intercalated sample $(S / P=0.96)$ than the $a-\mathrm{C}$ sample prepared at $800^{\circ} \mathrm{C}$ at low temperature $(1.3 \mathrm{~K})$. This change in magnetoresistance may be due to the dominance of electron-electron interaction or impurity scattering induced in the $a$-C system due to the sulfur incorporation. Figure 6 shows $B^{2}$ and

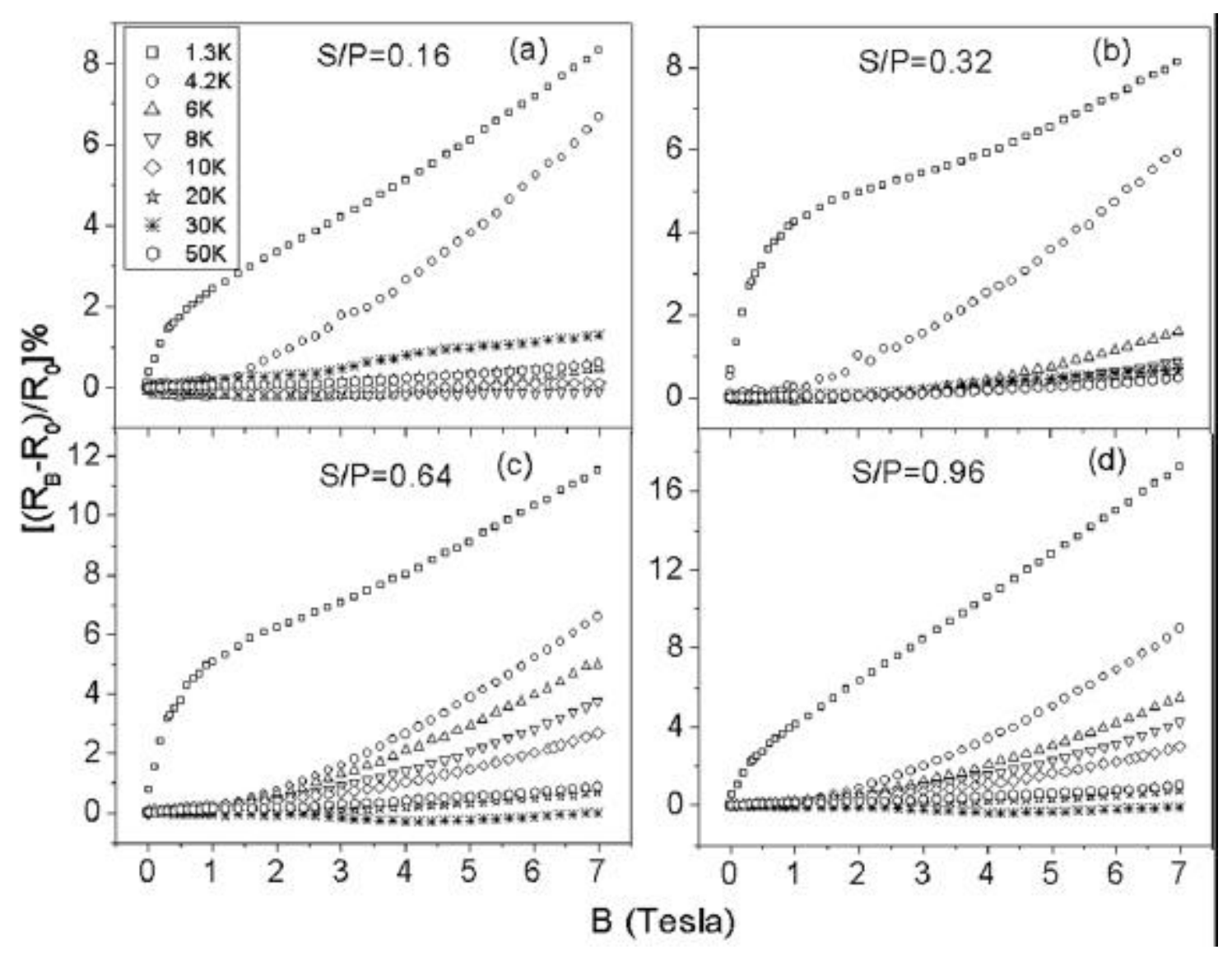

Figure 5. Magnetoresistance as a function of magnetic field at various temperatures for samples with (a) $S / P=0 \cdot 16$, (b) $S / P=0.32$, (c) $S / P=0.64$ and (d) $S / P=0.96$ pyrolysed at $800^{\circ} \mathrm{C}$. 
$B^{1 / 2}$ dependence of magnetoresistance at different temperatures for $a-\mathrm{C}: \mathrm{S}$ sample with $S / P=0.96$ prepared at $800^{\circ} \mathrm{C}$. As is seen in figure $6 \mathrm{a}$, magnetoresistance shows $B^{2}$ dependence over the entire field range $(0-7 \mathrm{~T})$ at all temperatures except $1.3 \mathrm{~K}$. It is attributed to the decrease in localization length in the presence of magnetic field as the system moves to insulating state (Dugdale 1995). Whereas the $B^{1 / 2}$ dependence of magnetoresistance (figure $6 \mathrm{~b}$ ) is observed only at high field regions and is independent of temperature.

\subsection{Thermal analysis}

Thermal stability of any intercalated system depends on the bonding between the host system and the guest species. XRD studies on $a$-C:S samples showed crystalline peaks corresponding to a sulfur embedded in the carbon
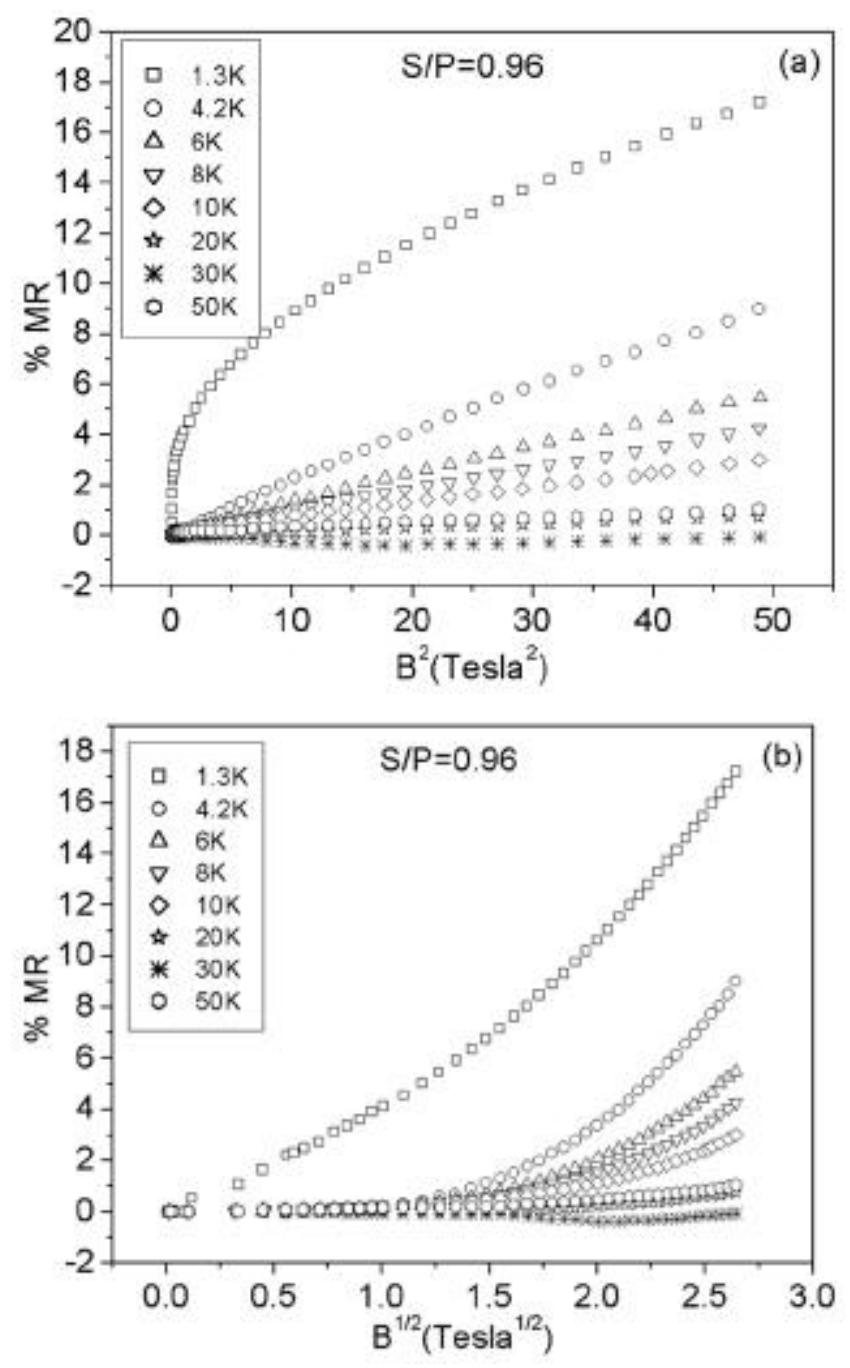

Figure 6. Magnetoresistance of $a-\mathrm{C}: \mathrm{S}$ sample with $S / P=0.96$ prepared at $800^{\circ} \mathrm{C}$ showing (a) $B^{2}$ dependence at low fields and (b) $B^{1 / 2}$ dependence at high fields. network, but the interconnection between carbon and sulfur atoms may be very weak. The intercalated sulfur may be present on the defects and discontinuities of the host structure. When these samples are subjected to thermal treatment, adsorbed sulfur can easily come out of the structure, as there are no strong bonds between the trapped sulfur and the carbon atoms. Even, XRD pattern of the $a$ $\mathrm{C}: \mathrm{S}$ sample annealed at $300^{\circ} \mathrm{C}$ in the inert atmosphere shows no crystalline peaks corresponding to sulfur, which thereby confirms the deintercalation of sulfur at that temperature. Figure 7 is the plot of thermogravimetry (TG) and differential TG (DTG) for samples with $S / P=0.32$ prepared at $800^{\circ} \mathrm{C}$ (figure $7 \mathrm{a}$ ) and $900^{\circ} \mathrm{C}$ (figure $7 \mathrm{~b}$ ). From the TG curve we can account for overall $22 \%$ and $43 \%$ weight loss for the samples prepared at $800^{\circ} \mathrm{C}$ and $900^{\circ} \mathrm{C}$, respectively. A sharp DTG peak at around $200^{\circ} \mathrm{C}$ corresponds to the deintercalation of sulfur from the $a-\mathrm{C}$ system, which accounts for a weight loss of 7-8\% for samples prepared at $800^{\circ} \mathrm{C}$, whereas for samples prepared at $900^{\circ} \mathrm{C}$ DTG peak is observed at $\sim 184^{\circ} \mathrm{C}$ associated with a $13-14 \%$ weight loss. This infers that the stability of the $a$-C:S system may decrease with the improving ordering, because
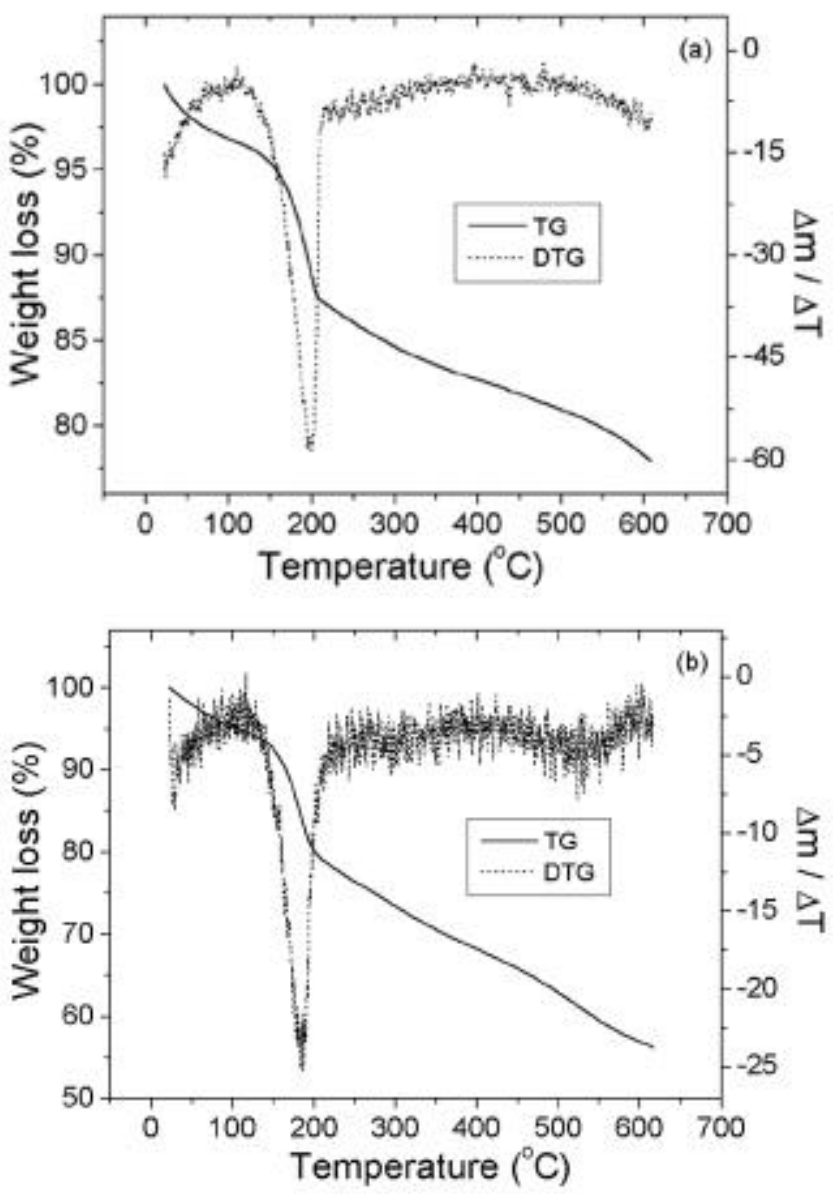

Figure 7. TG-DTG curves of $a$-C:S samples with $S / P=0 \cdot 32$ prepared at (a) $800^{\circ} \mathrm{C}$ and (b) $900^{\circ} \mathrm{C}$. 
$900^{\circ} \mathrm{C}$ prepared samples are more ordered and conducting than the samples prepared at $800^{\circ} \mathrm{C}$. Hence, with the increasing crystallinity or ordering, not only intercalation but also deintercalation seems easier. In the temperature range $200-600^{\circ} \mathrm{C}$, gradual weight loss is observed for both samples which can be attributed to the decomposition of carbon to form $\mathrm{CO}, \mathrm{CO}_{2}$. In this temperature range, sulfur that may be bonded to carbon atoms or may be trapped in the defects gets deintercalated and may also decompose to form oxides.

\section{Conclusions}

We have discussed the carbon-sulfur composite film preparation by vapour phase pyrolysis of maleic anhydride and sulfur. SEM and XRD studies depict the structural changes in the sulfur incorporated $a$-C films. Microhardness measurements conducted on the $a-\mathrm{C}: \mathrm{S}$ samples depict an increase in hardness with the increase in sulfur concentration. Increase in electrical conductivity is observed for the moderate sulfur intercalated samples, whereas for the higher sulfur concentration samples a drastic fall in conductivity is witnessed. Magnetoresistance measurement accounts for the drastic increase in the value of magnetoresistance for samples with high sulfur content and low pyrolysis temperatures. Thermal stability of $a-C: S$ samples is found to depend on the crystallinity or ordering of the system.

\section{Acknowledgements}

We are grateful to the Department of Science and Technology and the Council of Scientific and Industrial Research, New Delhi, for financial support.

\section{References}

Dugdale J S 1995 The electrical properties of disordered metals (Cambridge: Cambridge University Press)

Filik J, May P W, Pearce S R J, Wild R K and Hallam K R 2003 Diamond Relat. Mater. 12974

Gupta V, Mathur R B, Bahl O P, Tressaud A and Flandrois S 1995 Synth. Met. 7369

Gupta S, Martinez A, Weiner B R and Morell G 2002 Appl. Phys. Lett. 81283

Gupta S, Weiner B R and Morell G 2003 Appl. Phys. Lett. 83491

Hasegawa M, Takeuchi D, Yamanaka S, Ogura M, Watanabe H, Kobayashi N, Okushi H and Kajimura K 1999 Jpn. J. Appl. Phys. 38 L1519

Latha Kumari, Prasad V and Subramanyam S V 2003 Carbon 411841

Robertson J and O’Reilly E P 1987 Phys. Rev. B35 2946

Robertson J 1992 Phys. Rev. Lett. 68220

Robertson J 2002 Mater. Sci. Eng. R37 129

Sakaguchi I, Gamo M N, Kikuchi Y, Yasu E, Haneda H, Suzuki T and Ando T 1999 Phys. Rev. B60 R2139

Silva S R P, Carey J D, Khan R U A, Gerstner E G and Anguita J V 2002 Handbook of thin film materials (ed.) H S Nalwa (San Diego: Academic Press) Ch. 9, Vol. 4, p. 403 